\title{
Conservation and divergence of the histone code in nucleomorphs
}

\author{
Georgi K. Marinov ${ }^{*}$ and Michael Lynch
}

\begin{abstract}
Background: Nucleomorphs, the remnant nuclei of photosynthetic algae that have become endosymbionts to other eukaryotes, represent a unique example of convergent reductive genome evolution in eukaryotes, having evolved independently on two separate occasions in chlorarachniophytes and cryptophytes. The nucleomorphs of the two groups have evolved in a remarkably convergent manner, with numerous very similar features. Chief among them is the extreme reduction and compaction of nucleomorph genomes, with very small chromosomes and extremely short or even completely absent intergenic spaces. These characteristics pose a number of intriguing questions regarding the mechanisms of transcription and gene regulation in such a crowded genomic context, in particular in terms of the functioning of the histone code, which is common to almost all eukaryotes and plays a central role in chromatin biology.

Results: This study examines the sequences of nucleomorph histone proteins in order to address these issues. Remarkably, all classical transcription- and repression-related components of the histone code seem to be missing from chlorarachniophyte nucleomorphs. Cryptophyte nucleomorph histones are generally more similar to the conventional eukaryotic state; however, they also display significant deviations from the typical histone code. Based on the analysis of specific components of the code, we discuss the state of chromatin and the transcriptional machinery in these nuclei.
\end{abstract}

Conclusions: The results presented here shed new light on the mechanisms of nucleomorph transcription and gene regulation and provide a foundation for future studies of nucleomorph chromatin and transcriptional biology.

Keywords: Histones, Nucleomorphs, Histone code, Transcription, Chromatin

\section{Background}

A common trend in the evolution of endosymbiont genomes is their reduction [1, 2], best exemplified by organellar (chloroplast and mitochondrial) genomes in eukaryotes. This trend is also evident in the fate of secondary endosymbionts (i.e., eukaryotes that have become endosymbionts of other eukaryotes), which have arisen on several occasions in eukaryote evolution [3]. In most lineages bearing secondary plastids, all that remains from the eukaryotic endosymbiont is the plastid, with the nucleus having been lost. There are two notable exceptions to this rule, the chlorarachniophytes and the cryptophytes, which retain a remnant of the endosymbiont's nucleus and its genome in the form of a nucleomorph $[4,5]$.

*Correspondence: marinovg@iu.edu

Department of Biology, Indiana University, 47405 Bloomington, IN, United States
Strikingly, the chlorarachniophyte and the cryptophyte nucleomorphs do not share a common origin, having been acquired from a green and a red alga, respectively, yet their genomes have evolved convergently to a remarkably similar state [6, 7]. Nucleomorph genomes are the smallest eukaryote genomes known, typically just a few hundred kilobases in size; in all known cases, they are organized into three AT-rich chromosomes, with arrays of ribosomal RNA genes in their subtelomeric regions; they are extremely compacted, with almost nonexistent intergenic spaces (sometimes genes actually overlap), and even the genes themselves are frequently shortened [8-14]. These generalizations can be made thanks to the availability of fully sequenced nucleomorph genomes from several species: the chlorarachniophytes Bigelowiella natans [13] 
and Lotharella oceanica [12], and the cryptophytes Guillardia theta [9], Hemiselmis andersenii [14], Cryptomonas paramecium [10], and Chroomonas mesostigmatica [11].

While much has been learned from the analysis of these genome sequences, numerous important questions about nucleomorph biology remain unresolved. One such issue concerns the mechanisms of transcription and transcriptional regulation in such a crowded and compact genomic environment. All steps of the transcriptional cycle, as well as virtually every aspect of gene regulation in eukaryotes, involve the participation of the histone proteins around which DNA is packaged, usually through their posttranscriptional modifications (PTMs). PTMs are deposited in a regulated manner, most often (but not only) in the unstructured $\mathrm{N}$-terminal tails of histones. They serve as recruitment platforms for effector proteins, which have specific recognition domains for certain marks, thus generating the so called "histone code" [15]. Hundreds of histone modifications have been identified [16] and while most of them are yet to be functionally characterized in detail, the role of a significant number of histone marks is well understood. These include specific marks associated with transcription initiation, transcription elongation, the formation of repressive heterochromatin, mitotic condensation of chromosomes, and other processes [17]. Importantly, most of the posttranscriptionally modified residues are deeply conserved among the majority of eukaryotes [18], implying the ancient origins the histone code and the major functional importance of each individual histone mark.

The extent to which these modifications are conserved in nucleomorphs may provide valuable insights into their biology. Some of the open questions are: 1) whether transcription initiation proceeds through similar mechanisms as in conventional eukaryote nuclei, where the one or two nucleosomes around the transcription start site acquire a specific set of modifications [19] (the tightly packed nucleomorph genes simply do not provide sufficient space for this arrangement given the $\sim 146 \mathrm{bp}$ minimal footprint of the nucleosome); 2) whether the complex cycle of histone modifications and nucleosome displacement associated with transcription elongation by RNA Polymerase II [20-22] is conserved and operates over the very short nucleomorph genes; 3 ) whether repressed heterochromatin, which is associated with telomeres, pericentromeric areas, and considerable portions of the rest of the genome in many eukaryotes [23-25] exists in nucleomorphs; 4 ) whether the mechanisms for regulating chromosome condensation during cell division [26] are conserved in nucleomorphs; 5) whether the patterns of conservation and divergence from the common eukaryotic state are as convergent between chlorarachniophyte and cryptophyte nucleomorphs as are so many other features of their genomes and biology.
This study addresses these questions by analyzing the sequence of nucleomorph histone proteins and the conservation of the key amino acid residues involved in these processes. Chlorarachniophyte nucleomorph histones appear to have lost almost all major histone modifications that are core to the functioning of the histone code. Cryptophyte nucleomorph histones have retained the capacity for a larger number of the key chromatin marks, with the exception of Cryptomonas paramecium, whose histone proteins are most divergent within that group. In addition, in all species (both in chlorarachniophytes and in cryptophytes), the heptad repeats in the C-terminal domain (CTD) tail of the largest subunit of RNA Polymerase II, which play a key role in many aspects of the transcriptional cycle and mRNA processing [27, 28], are absent. These results suggest that the transcriptional machinery in nucleomorph exists in a highly derived state relative to its ancestral eukaryotic condition.

\section{Results}

\section{Histone genes in nucleomorphs}

Histone genes were identified in nucleomorph assemblies using a combination of HMMER domain scans $[29,30]$ and BLASTP [31] searches. It has been previously shown that in Bigelowiella natans histones $\mathrm{H} 2 \mathrm{~A}$ and $\mathrm{H} 2 \mathrm{~B}$ are encoded in the host nucleus and imported into the nucleomorph [32]. Consistent with these reports and the existing annotations, only histones $\mathrm{H} 3$ and $\mathrm{H} 4$ can be detected in both chlorarachniophyte nucleomorph genomes. In contrast, all cryptophyte nucleomorphs also encode a version of $\mathrm{H} 2 \mathrm{~B}$, implying that only $\mathrm{H} 2 \mathrm{~A}$ has been transferred to the host nucleus in this lineage. Nucleomorph histones exhibit unique patterns of divergence relative to conventional nuclear histones as evident from their phylogenetic relationships (Additional file 1: Figures S1, S2, S3 and S4). No apparent linker histone genes were found in any of the nucleomorphs.

To gain insight into their functional properties, multiple sequence alignments of nucleomorph histones were carried out, using nuclear core histones from several other eukaryotes, in which the role of histone marks in chromatin biology has been extensively studied, as reference. Figures 1 and 2 show these alignments for chlorarachniophyte histones $\mathrm{H} 3$ and $\mathrm{H} 4$. In both species, a significant portion of the $\mathrm{N}$-terminal tail of histone $\mathrm{H} 3$ (between residues 10 and 38) is missing, and overall the sequence is highly divergent. Surprisingly, the Bigelowiella histone $\mathrm{H} 3$ has a 10-amino acid insertion in the core histone part of the protein, which is not shared with Lotharella; its significance is not clear at present. The properties of H4 histones are similar: both species share a $\sim 10$-amino acid deletion at the very end of the $\mathrm{N}$-terminal tail and the sequences are highly divergent from those of other eukaryotes. 


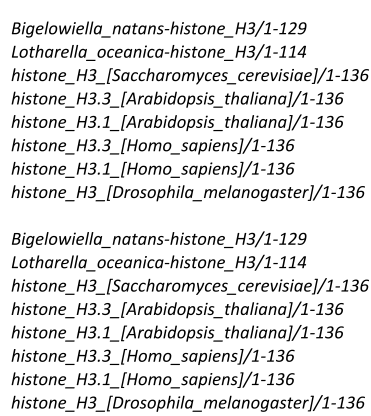

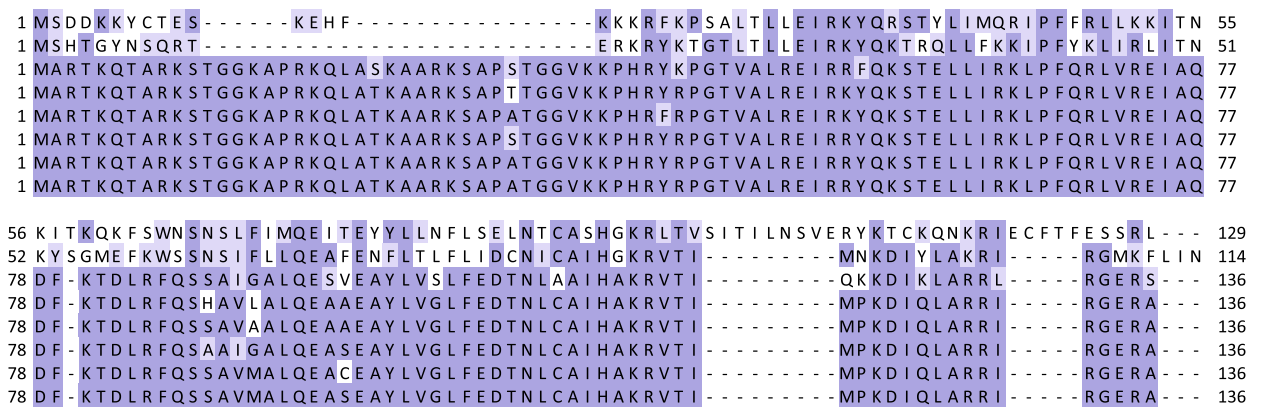

Fig. 1 Multiple seqience alignment of chlorarachniophyte nucleomorph histones $\mathrm{H} 3$ and nuclear $\mathrm{H} 3$ histones from several representative eukaryotes. Multiple sequence alignments were carried out using MUSCLE [102] and visualized using Jalview [103]

Cryptophyte histone $\mathrm{H} 3$ sequences are generally much more similar to conventional eukaryotic histone H3 than those of chlorarachniophytes, yet they also exhibit numerous unconventional features (Fig. 3). The extreme $\mathrm{N}$-terminal end is particularly well conserved, with the exception of that of Cryptomonas's H3, yet all species display significant sequence divergence roughly coinciding with the deleted region in chlorarachniophyte $\mathrm{H} 3$ proteins, including a large insertion in Chroomonas.

Chroomonas also has large insertions in the $\mathrm{N}$-terminal tail of its histone H4 (Fig. 4) though the rest of the sequence is relatively similar to that of other eukaryotes, as are those of the other three cryptophytes.

Unlike chlorarachniophytes, cryptophytes also have a nucleomorph-encoded histone $\mathrm{H} 2 \mathrm{~B}$ protein. It should be noted that higher levels of sequence divergence are observed for $\mathrm{H} 2 \mathrm{~A}$ and $\mathrm{H} 2 \mathrm{~B}$ proteins within eukaryotes in general. Nevertheless, their conservation characteristics in cryptophytes are similar to those of cryptophyte $\mathrm{H} 3$ and $\mathrm{H} 4$ nucleomorph histones (Additional file 1: Figure S5), with significant divergence seen in the $\mathrm{N}$-terminal tail (which is completely missing in Cryptomonas and Guillardia, and has large deletions in Chroomonas and Hemiselmis).

\section{The histone code in nucleomorphs}

To assess the conservation of the histone code in nucleomorphs, the preservation of modified residues and their immediate three- and five-amino acid sequence contexts was analyzed. There are some limitations to this approach. While the conservation of a given residue does not necessarily mean that its post-translational modifications are also conserved, its absence does mean that the corresponding modifications have been lost. In addition, sequence context conservation can be due to the presence of independent constraints on the residue and its neighborhood rather than because of conservation of its posttranscriptional modifications. Nevertheless when observed, such conservation makes it more plausible that the capacity for depositing the modification has been retained. The conservation of the best functionally understood residues on histones $\mathrm{H} 3$ and $\mathrm{H} 4$ in chlorarachniophyte and cryptophyte nucleomorphs as evaluated according to these criteria is shown in Fig. 5.

\section{Histone marks associated with heterochromatin}

In most eukaryotes, heterochromatin occupies the pericentromeric and telomeric regions of chromosomes and is also used as a mechanism for silencing transposable elements as well as some host genes. Histone marks play a central role in the formation of heterochromatin, in particular the trimethylation of histone 3 lysine 9 (H3K9me3), which classically serves as a recruitment platform for the HP1 heterochromatin protein [33-35]. Other marks

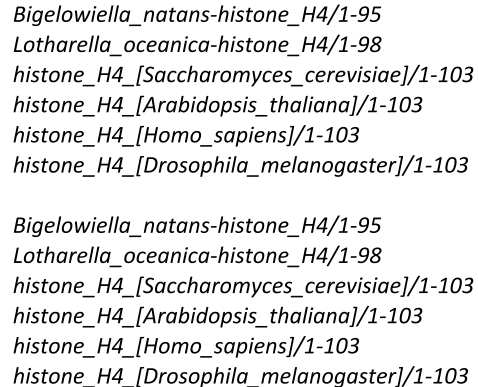

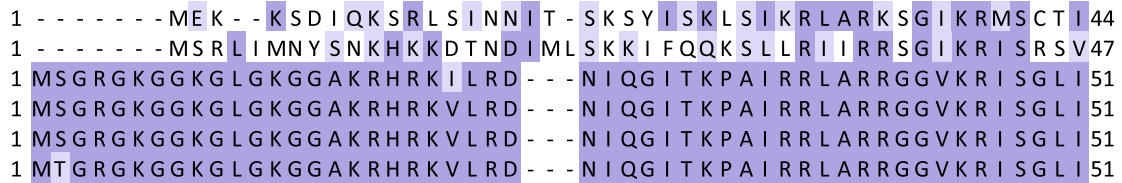

45 YAE I NKF I VEFLTKIVKD I I I FCRYENRT L I KVSDVLVVLRRYGKMYYG - KR 48 Y I ELNS I I KKFLLK I VKDTLIVC I YNHRK I I TASDVIFSLKRNGQN I YG-GL 52 Y EEVRAVLKSFLESVIRDSVTYTEHAKRKTVTSLDVVYALKRQGRTLYGFGG 52 Y EETRGVLKIFLENVI RDAVTYTEHARRKTVTAMDVVYALKRQGRTLYGFGG 52 YEETRGVLKVFLENVI RDAVTYTEHAKRKTVTAMDVVYALKRQGRTLYGFGG 52 YEETRGVLKVFLENVI RDAVTYTEHAKRKTVTAMDVVYALKRQGRTLYGFGG

Fig. 2 Multiple seqience alignment of chlorarachniophyte nucleomorph histones $\mathrm{H} 4$ and nuclear $\mathrm{H} 4$ histones from several representative eukaryotes. Multiple sequence alignments were carried out using MUSCLE [102] and visualized using Jalview [103] 


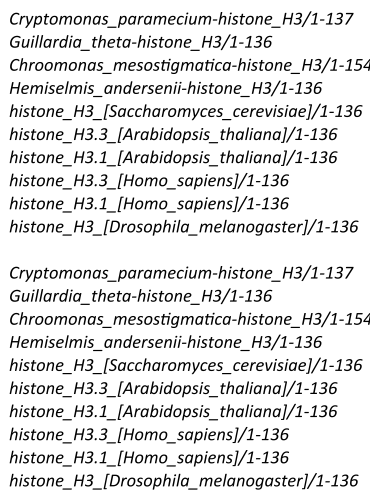

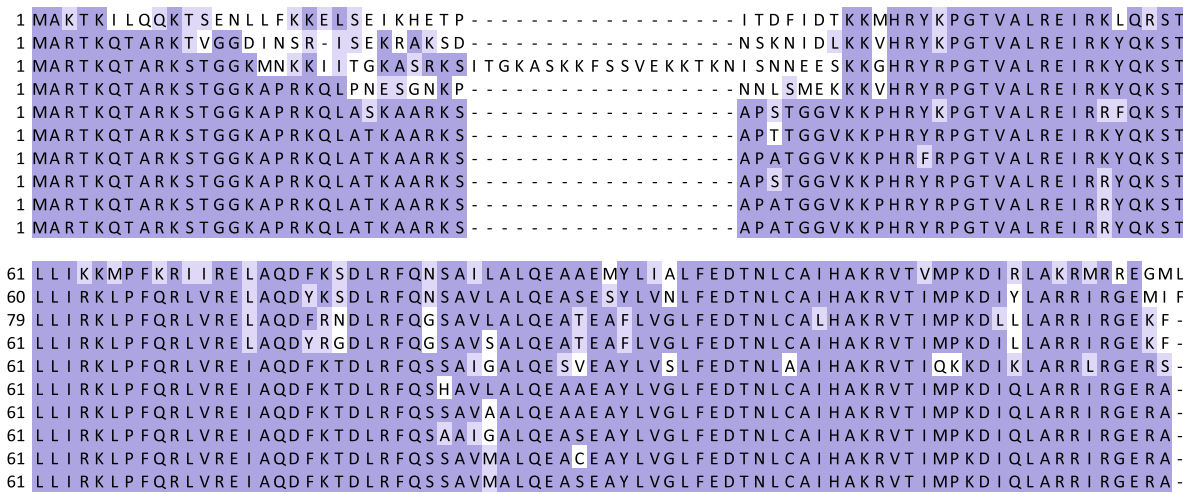

FHRYPTVALRE I RKLQRSTD 60 60
59 59
78 60 60

Fig. 3 Multiple seqience alignment of cryptophyte nucleomorph histones $\mathrm{H} 3$ and nuclear $\mathrm{H} 3$ histones from several representative eukaryotes. Multiple sequence alignments were carried out using MUSCLE [102] and visualized using Jalview [103]

associated with heterochromatin include H4K20me3 [35, 36] and H3K64me3 [37]. In addition, another repressive mechanism operates through the H3K27me3 mark, which is key to Polycomb complex-mediated gene repression in both plants and metazoans [38, 39]. It should be noted that different combinations of marks might be associated with distinct types of heterochromatin in multicellular eukaryotes [40, 41].

Strikingly, H3K9 and H3K27 are completely absent in chlorarachniophyte nucleomorphs. H4K20 and H3K64 are observed in Lotharella, but their sequence context is not conserved.

In cryptophytes, H3K9 and its sequence context are conserved in Chroomonas and Hemiselmis; H3K9 is present in Cryptomonas and Guillardia, but the sequence context is not conserved. H3K27 is absent from Cryptomonas and Guillardia, and present in Chroomonas and Hemiselmis, but without the context being conserved. H3K64 is well conserved in cryptophytes with the exception of Cryptomonas, while H4K20 is present in all species but its context is perfectly conserved only in Hemiselmis.

\section{Phosphorylation marks associated with chromosome dynamics during mitosis}

Histone proteins contain multiple phosphorylation sites, which play an important role in the regulation of the dynamics of chromosome condensation during mitosis. These include H3T3ph [42-44], H3S10ph [45-48], H4S1ph [49], and others [50-53]. In addition, H3S28ph is associated with transcriptionally active chromatin $[54,55]$.

These marks seem to be largely absent from chlorarachniophyte histones (H3T3 is present in Lotharella and H3S10 in Bigelowiella, but no sequence context conservation is observed; $\mathrm{H} 4 \mathrm{~S} 1$ is absent from both species).

Chroomonas, Guillardia and Hemiselmis retain H3T3, H3S10 is conserved in Chroomonas and Hemiselmis but absent from the more divergent tails of Cryptomonas and Guillardia. H4S1 is also absent from cryptophytes.

H3S28 is nearly absent from all nucleomorphs.

\section{Histone marks associated with the transcription elongation cycle}

The process of transcription faces a major barrier in eukaryotes in the form of the nucleosome, which

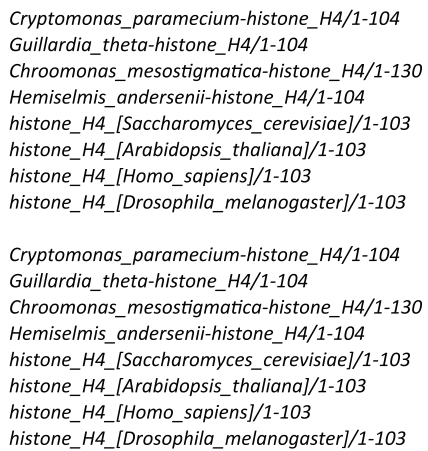

Cryptomonas_paramecium-histone_H4/1-104 Guillardia_theta-histone_H4/1-104 Chroomonas_mesostigmatica-histone_H4/1-130 Hemiselmis_andersenii-histone_H4/1-104 histone H4_[Saccharomyces_cerevisiae]/1-103 histone_H4_[Arabidopsis_thaliana]/1-103 histone_H4_[Homo_sapiens]/1-103 histone_H4_[Drosophila_melanogaster]/1-103

Cryptomonas_paramecium-histone_H4/1-104 Guillardia_theta-histone_H4/1-104 Chroomonas_mesostigmatica-histone_H4/1-130 Hemiselmis_andersenii-histone_H4/1-104 histone H4_[Saccharomyces_cerevisiae]/1-103 histone_H4_[Arabidopsis_thaliana]/1-103 histone_H4_[Homo_sapiens]/1-103 histone_H4_[Drosophila_melanogaster]/1-103

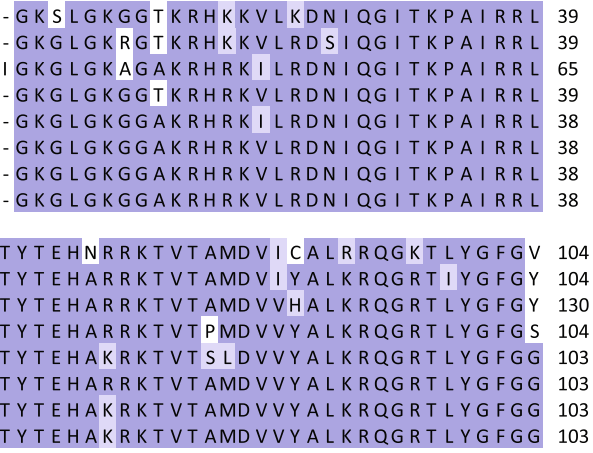

Fig. 4 Multiple seqience alignment of cryptophyte nucleomorph histones $\mathrm{H} 4$ and nuclear $\mathrm{H} 4$ histones from several representative eukaryotes. Multiple sequence alignments were carried out using MUSCLE [102] and visualized using Jalview [103] 


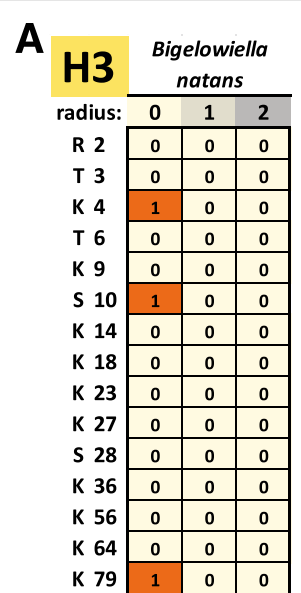

B

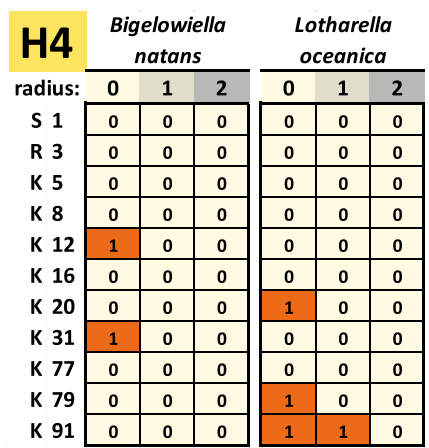

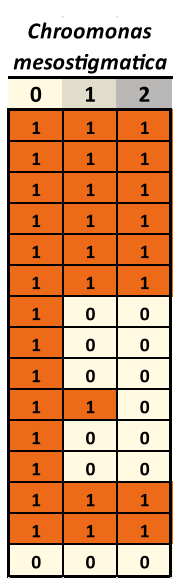

Chroomonas

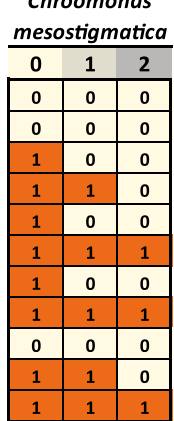

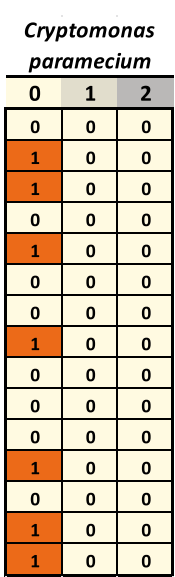

Cryptomonas

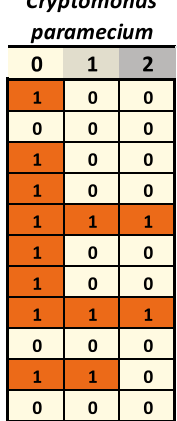

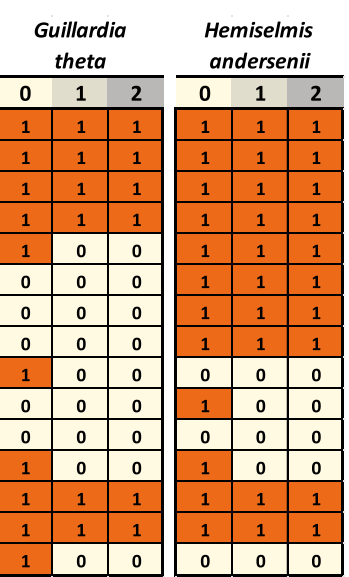

Guillardia

Hemiselmis

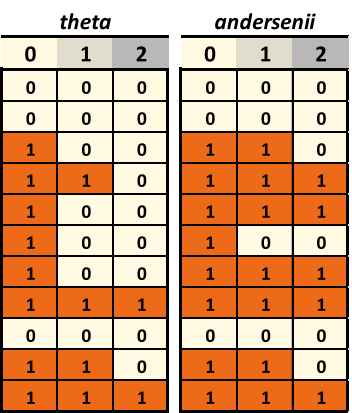

Fig. 5 Conservation of key posttranscriptionally modified residues in chlorarachniophyte and cryptophyte nucleomorph histones $\mathrm{H} 3$ and $\mathrm{H} 4$. a Histone $\mathrm{H} 3$; $\mathbf{b}$ Histone $\mathrm{H} 4$. The radius $r$ refers to the size of the context considered when scoring conservation. When $r=1$, only the residue itself is considered; when $r=0$, a perfect match to the three-amino acid peptide also including the flanking residues on each side is required; when $r=2$, the five-amino acid peptide also including the two flanking residues on each side is considered. A score of 1 means conservation according to these criteria, while a score of 0 means lack of conservation. Conservation was scored against the Homo sapiens histone H3.3 and H4 sequences

inhibits transcriptional elongation. A complex cascade of molecular events, which involves a number of histone modifications, has evolved in response to this constraint. Acetylation marks are deposited on nucleosomes in front of the elongating polymerase, in order to facilitate the opening of chromatin. The FACT complex $[56,57]$ plays the role of a histone chaperone, partially disassembling nucleosomes so that the polymerase can transcribe through them, then reassembling them. This process also involves the monoubiquitination of histone $\mathrm{H} 2 \mathrm{~B}$ at a specific site (H2BK120ub1 $[58,59])$. Transcriptional elongation is also associated with the deposition of H3K36me3 $[60,61]$, which plays an important role in recruiting histone deacetylases after the polymerase has passed [62-64] so that the newly deposited acetylation marks can be removed and potential cryptic transcription repressed [65]. Methylation of H3K79 is also associated with transcriptional elongation [66-69]. Finally, in addition to its role in elongation, H3K36me3 seems to also be involved in the regulation of splicing and RNA processing [70-75].

Remarkably, H3K36 is completely absent in chlorarachniophytes, and is present, but with a poorly conserved sequence context, in cryptophytes. H3K79 is observed in Bigelowiella, Cryptomonas, and Guillardia, and its sequence context is moderately conserved, but it is absent from all other species. H2BK120 is present in all cryptophytes (Additional file 1: Figure S6), but without any conservation of the sequence context.

\section{Histone marks associated with promoters}

The classical marker of eukaryotic promoters is the deposition of H3K4me3 [76, 77]. A lysine residue is present at that position in Bigelowiella, but its sequence context is very different, and it has been replaced with a glycine in Lotharella.

The three cryptophytes with relatively well-conserved $\mathrm{N}$-terminal tails do possess a correspondingly wellconserved H3K4, the exception being the highly divergent Cryptomonas tail.

\section{Histone marks associated with activation/repression of transcription}

A number of histone marks have been described as having a positive or negative correlation with transcription, 
either by regulating the modification of other residues (what is often referred to as histone mark "crosstalk"), by generally serving to create a more open chromatin state (acetylation being the primary example), or through other less well understood means. These include methylation on several arginines (H3R2me2 [78-80], H4R3me2 [81, 82]), which can have positive or negative effects on transcription depending on whether the dimethylation is symmetric or asymmetric, H3T6ph [83] and acetylation marks with generally activating effects (H3K14ac, H3K18ac, H3K23ac, H4K5ac, H4K8ac, H4K12ac, H4K16ac, H4K31ac, H2BK5ac, H2BK12ac, H2BK15ac, H2BK20ac, and others), as well as acetylation marks with better understood specific roles (for example, H3K56ac [84-86] and H4K91ac [87], which might also play a role in nucleosome assembly in addition to gene regulation).

All of these residues are either completely missing or poorly conserved in chlorarachniophytes, but display higher levels of conservation in cryptophytes (with the Cryptomonas being the exception).

\section{Absence of RNA Polymerase II CTD heptad repeats in nucleomorphs}

The histone code is not the only code playing a major role in transcriptional elongation. It operates in concert with the so called CTD code [88], consisting of the posttranscriptional modifications of the C-terminal domain tail of the largest subunit of RNA Polymerase II (Rpb1). Ancestrally to all extant eukaryotes [89], the CTD domain contains a large number of repeats of the consensus heptad sequence YSPTSPS [90] (note that the repeats are not always perfect matches to the consensus). The heptad contains five phosphorylation sites and two proline isomerization sites. Specific modifications are deposited on the tail during each phase of the transcriptional cycle, and serve to recruit various effector and regulatory proteins $[27,28]$. As a result the CTD repeats play a pivotal role in almost all aspects of transcriptional elongation and mRNA processing, and their status in nucleomorphs is of significant interest.

All six nucleomorphs encode an Rpb1 protein. However, all of these proteins completely lack heptad repeats (Additional file 1: Figure S7) indicating a corresponding absence of conservation of the CTD code.

\section{Discussion}

The analysis of nucleomorph histone protein sequences presented here reveals a number of puzzling and potentially fascinating aspects of nucleomorph chromatin biology.

The first major observation is that while nucleomorph histones are generally quite derived in all species, this is much more so in chlorarachniophytes than in cryptophytes, with most of the classic sites of histone modification completely missing in the former, and significantly higher conservation observed in the latter. However, even within cryptophytes one species, Cryptomonas paramecium, also exhibits a significant divergence from the conventional state and the loss of most key histone mark residues. The evolutionary forces and biochemical constraints responsible for these patterns remain to be elucidated by future studies.

Second, it seems that typical heterochromatin is most likely absent from nucleomorphs given that the key residues participating in its formation have been lost. This is not entirely surprising - the extremely small size, compact nature and high transcriptional activity [91] of nucleomorph chromosomes presumably eliminates the need for heterochromatin, even in telomeric regions.

A similar relaxation of biochemical constraints might be behind the loss of the phosphorylation sites involved in chromosome condensation during mitosis. The mechanisms of nucleomorph division, its regulation, as well as the chromosome dynamics during the process, remain very poorly understood, so future studies will have to bring more light onto this subject. Still, it is conceivable that nucleomorph chromatin is not compacted in a fashion similar to that of autonomous eukaryote nuclei during mitosis.

The most striking loss of functionality in nucleomorphs is associated with transcription initiation and elongation. The potential for initiation-associated methylation of H3K4 at least exists in cryptophytes, but is certainly lost in chlorarachniophytes. The mechanisms for promoter definition and the details of transcription initiation in this context are at present a mystery. The situation is even more puzzling when it comes to transcriptional elongation. It is possible that H3K79 methylation is happening in cryptophytes, and H3K36 methylation and H2BK120 ubiquitination cannot be confidently ruled out, but they are almost certainly completely absent in chlorarachniophytes. Equally striking is the absence of heptad repeats from the tail of the RNA Polymerase II largest subunit. In total, these observations suggest that both the nucleosome dynamics during transcriptional elongation and the coupling of transcription with mRNA processing have evolved to a highly derived state in nucleomorphs. One possibility is that the high transcriptional activity of nucleomorphs allows for a permanently open nucleosome state; however, it is not clear how cryptic transcription can be prevented if that is the case, and such a hypothesis only explains the absence of H3K36me3, but not that of Rpb1's CTD repeats, which are crucial for a wide variety of other processes in addition to the acetylation/deacetylation cycle during elongation. 
The poor conservation of many of the residues known primarily for being acetylated (and not yet linked to specific functions beyond that) is also not entirely surprising, as the role of opening chromatin can probably be taken over by other lysine residues (indeed, studies in yeast in which lysine residues have been systematically mutated have shown substantial redundancy between acetylation sites [92, 93]). Histone acetylation is almost certainly happening in nucleomorphs, as all six nucleomorph genomes encode a single histone deacetylase protein, histone acetyltransferases are detected in all cryptophyte nucleomorphs (Additionalf file 1: Figure S8), and additional ones might be imported from the host (although it is possible that they are retained because of their importance for the acetylation of non-histone proteins and not of histones themselves). Unfortunately, the problem of identifying the complete set of readers and writers of epigenetic marks in nucleomorphs is currently quite difficult because it is not known how many and which proteins are imported from the host. Thus while the presence of a given protein in the nucleomorph genome confirms its presence in the nucleomorph, its absence from the genome does not necessarily mean that it is not imported from the host; the histone genes themselves are a prime example of the ubiquity of this phenomenon.

Histone mark readers, writers, and erasers include the Dot1 methyltransferase, which methylates H3K79, no examples of which are found in nucleomorphs genomes (Additional file 1: Figure S8); SET domain proteins, which deposit all other lysine methylations [94], two of which are found in Chroomonas, Guillardia, and Hemiselmis nucleomorphs, but none in Cryptomonas paramecium and the chlorarachniophytes; histone acetyltransferases (HATs) [95] (found in cryptophyte nucleomorph genomes but not in chlorarachniophytes); the sirtuin deacetylases [96] (also not present in any of the species); bromodomain-containing proteins, which read acetylation marks [97] (found in some cryptophyte nucleomorphs); chromodomain-containing proteins, which read lysine methylated residues [98] (none found in nucleomorphs); PHD finger proteins, readers of lysine and arginine methylations [99] (also not present), and the Jumonji and LSD1 families of lysine demethylases [100] (not present in nucleomorphs either). A putative SWI/SNF chromatin remodeling protein is identified in most nucleomorphs, as is a Hira histone chaperone [101] in both cryptophytes and Bigelowiella as well as an ASF1 histone chaperone [101] in cryptophytes (Additional file 1: Figure S8). No FACT homologs are identified, and neither are DNA methyltransferases or other typical eukaryotic DNA methylation-related proteins (methylbinding domains), which read DNA methylation in eukaryotes.

\section{Conclusions}

Overall, the observations presented here suggest that a substantial fraction of the epigenomic toolkit has been lost from nucleomorphs (consistent with the divergence of their histone sequences) and much of whatever remains of it has been transferred to the nucleus, as has happened to many other genes previously resident in the nucleomorph genome. The loss of the code has most likely happened in concert with a loss of at least some of the chromatin remodeling and regulation functionality that it is normally a part of. However, how much exactly has been lost remains to be determined by future experiments. Given that some of the processes concerned are fundamental to the biology of transcription in eukaryotes, it is not easily envisioned how they could have been lost. One tantalizing possibility is that even if much of the conventional histone code has been lost, substantial innovation has occurred in parallel, and other residues have assumed the roles previously played by the classical modified residues known from autonomous nuclei. The several large insertions in histone proteins observed in Chroomonas, containing a number of lysine residues, lending some support to such an idea.

Future studies utilizing the power of modern proteomics and functional genomics should provide insight into these questions, by identifying the post-translational modifications of nucleomorph histones, and by mapping the chromatin structure, the distribution of modified histones, and the positions and identities of regulatory and transcriptional elements in nucleomorph genomes.

\section{Methods}

Except where otherwise stated, all analyses were performed using custom-written python scripts.

Nucleomorph genome sequences were obtained from the NCBI nucleotide database (GenBank: DQ158858.1, GenBank: DQ158857.1, GenBank: DQ158856.1, GenBank: CP003682.1, GenBank: CP003681.1, GenBank: CP003680.1, GenBank: CP002174.1, GenBank: CP002173.1, GenBank: CP002172.1, GenBank: NC_002753.1, GenBank: NC_002752.1, GenBank: NC_002751.1, GenBank: CP000883.1, GenBank: CP000881.1, GenBank: CP000881.1, GenBank: CP006629.1, GenBank: CP006628.1, GenBank: CP006627.1).

Histone genes were annotated using a combination of HMMER3.0 [29] scans against the Pfam 27.0 database [30] (scanning for histone fold domains) and BLASTP [31] searches (using histone sequences from Homo sapiens, Saccharomyces cerevisiae, Drosophila melanogaster, and Arabidopsis thaliana as queries). Multiple sequence alignments were carried out using MUSCLE [102] and visualized using Jalview [103]. 
Chromatin modification and remodeling proteins were identified using HMMER3.0, with an e-value cutoff of $10^{-8}$, as well as an additional BLAST search using chromatin proteins from Homo sapiens, the red alga Cyanidioschyzon merolae and the green algae Chlamydomonas reinhardtii and Micromonas pusilla as queries.

\section{Reviewers' comments}

Lakshminarayan lyer (Reviewer 1)

Marinov and Lynch provide a useful analysis of the nucleomorph histone code using the existing completely sequenced nucleomorph genomes. By comparing histone sequences, the study brings into focus the unique aspects of transcriptional/chromatin regulation in these nucleomorphs. In particular, one can posit what type of modifications are likely to be retained in these reduced genomes, of which some might be provided from their eukaryotic hosts. The sequence analysis of the histones is thorough. I do have a few additional pointers that pertains to the discussion and supplementary Figure 4. Comments: 1. The list of domains involved in post-translational modifications that were analyzed isnt complete (For other domains including readers and modifiers see PMIDs: $21507350,21507349,19092802)$. It would be prudent to check domains such as CXXC, LSD1, SAD/SRA and so on. 2. A word of caution: some PTM proteins and readers can diverge, so searches with individual proteins as starting points in addition to running automatic profiles should be done. For example, I detected a GCN5 acetyltransferase in the Guillardia theta nucleomorph (gi: 162606188) possessing both the acetylase and bromo domains. This is in line with the conservation of lysine residues in $\mathrm{H} 3$ such as H3K9, H3K14 and H3K23 that are substrates for this acetylase, in this nucleomorph. I have not checked the other nucleomorph genomes, but it might be prudent to check individual genomes carefully for divergent homologs.

Authors response: Thank you for you suggestions. The analysis of chromatin modifying and remodeling proteins has been updated accordingly.

\section{Berend Snel (Reviewer 2)}

\section{Reviewer summary}

The authors present an in depth analysis of the alignments of the histone proteins of nucleomorph genomes to reveal the evolutionary fate of the histone code of these genomes. Although the histone residues provide interesting and relevant discussion, I missed certain analyses and information which I think should be added. Moreover the results are somewhat limited as it basically consists of 2 alignments with some textual description of histone modifying enzymes, and without additional phylogenetic analysis.

\section{Reviewer recommendations to authors}

Upon first reading the manuscript quite some relevant information which at least for me was necessary to understand the story was missing or only presented in the discussion. Specifically the following issues are I think unclear or missing but needed for context or as results. First that the machinery for depositing relevant histone marks is needed to modify the histones, and to what extent this machinery is present on the nucleomoprh genome or the host nuclear genome. And if it is present on the nuclear genome, to what extend these proteins might or might not be targeted to the nucleomoprh nucleus. These points are to a certain extent present in the discussion, but part of these points are results and should be in the results (e.g. the histone modification enzymes encoded by the nuclear host genome as discussed on page 8 ). Other points are not sufficiently discussed at all while they are relevant for the interpretation. I specifically think that the manuscript insufficiently discusses what we know about proteins being translocated to the nucleomorph. What signals are present in sequences being targeted to the nuceomorph and if bioinformatics or proteomics can or has helped in identifying such proteins.

Authors response: The reasons we did not do a thorough analysis of the nuclear genomes are that first, we only have nuclear genome sequences for one chlorarachniophyte (Bigelowiella) and one cryptophyte species (Guillardia), thus there was no way to generalize results over the whole of each group, and second and more important, in silico nucleomorph targeting predictions are very far from being reliable. Direct proteomic measurements exist only for the Bigelowiella natans nucleomorph [104] and that particular study only identified 324 proteins. Of those, only half had predicted SP and/or TP targeting sequences, and the imported histones and transcription factors had no obvious such sequences at all.

After reading the manuscript I was convinced that the nucleomorph histones are indeed quite diverged but this information cannot be fully interpreted if its (predicted) proteomic context is not at least partially provided. Related to the previous point. Obviously the nuclear host genome can also encode proteins of nucleomorph origin. And some of these might still be active in the nucleomorph cytoplasm or nucleus. In addition, the nucleomorph encoded histones of especially the chlorarachniophyte are so diverged I was wondering if they are in fact even orthologous to $\mathrm{H} 3$ or $\mathrm{H} 4$. Both of these issues could perhaps be clarified by constructing gene trees (although that is exceedingly difficult for histone proteins and especially for such highly diverged proteins). Nevertheless it would be very interesting if such a gene tree would show for example a host nuclear encoded protein in Bigelowiella that is closely related to the green algae. Even in the absence of any firm biochemical knowlegde on 
the transfer mechanism such a gene would provide good candidates for a potential histone protein that could be active in the nucleomorph nucleus and could "rescue" the diverged nucleomorph encoded protein. A quick panther scan of the Bigelowiella genome with the $\mathrm{H} 3$ profile finds 9 predicted histone $\mathrm{H} 3$ proteins. This number indeed raises the question of paralogy, orthology and orther "pseudo" orthologs originated by nuclear transfers of secondary endosymbiosis.

Authors response: We did not initially carry out a phylogenetic analysis of all Bigelowiella and Guillardia histone proteins because that has been previously done in Hirakawa et al. 2011, while our focus was on the functional inferences we can generate based on the sequences, as a starting point towards a future experimental dissection of that functionality. An updated such analysis will be of great interest in the future when more nuclear genomes become available, but at the moment there are only these two. We have included phylogenetic trees of the relevant sequences using transcriptome assemblies from the Marine Microbial Eukaryote Transcriptome Sequencing Project (MMETSP) [105] as supplementary figures in the revised manuscript.

\section{Minor issues}

I do not understand why the alignments in Figs. 1, 2 and 3 are presented in the way they are. i.e. why is the for example the alignment of reference eukaryote $\mathrm{H} 3$ with histone $\mathrm{H} 3$ of Bigelowiella a separate alignment from that of Lotharella? I see no reason why this could not be a single alignment. Normally I would also expect the cryptophyte histones to be in a single alignment with the reference species.

Authors response: The figures have been modified according to the reviewer's suggestions.

\section{Additional file}

Additional file 1: Supplementary Figures. (PDF 7127 kb)

\section{Abbreviations}

A: adenine; CTD: C-terminal domain; HAT: histone acetyltransferase; MMETSP: Marine Microbial Eukaryote Transcriptome Sequencing Project; PTM: posttranscriptional modification; RNA: deoxyribonucleic acid; RNA: ribonucleic acid; SP: signal sequence; T:thymine; TP: transit peptide sequence.

\section{Competing interests}

The authors declare that they have no competing interests.

\section{Authors' contributions}

GKM conceived and designed the study. GKM analyzed the data. GKM and ML wrote the manuscript. Both authors read and approved the final manuscript.

\section{Acknowledgements}

Support was provided by National Science Foundation awards MCB-1050161 and NSFMCB-1518060. This material is also based upon work supported by the National Science Foundation grant CNS-0521433.

Received: 11 December 2015 Accepted: 22 March 2016

Published online: 05 April 2016

\section{References}

1. Blanchard JL, Lynch M. Organellar genes: why do they end up in the nucleus? Trends Genet. 2000;16(7):315-20.

2. Moran NA, Bennett GM. The tiniest tiny genomes. Annu Rev Microbiol. 2014;68:195-215.

3. Keeling PJ. The endosymbiotic origin, diversification and fate of plastids. Philos Trans R Soc Lond B Biol Sci. 2010;365(1541):729-48.

4. Greenwood AD. The Cryptophyta in relation to phylogeny and photosynthesis In: Sanders JV, Goodchild DJ, editors. Electron microscopy 1974. Canberra: Australian Academy of Sciences; 1974. p. 566-67.

5. Greenwood AD, Griffiths HB, Santore UJ. Chloroplasts and cell compartments in Cryptophyceae. Brit Phycol J. 1977;12:119.

6. Archibald JM. Nucleomorph genomes: structure, function, origin and evolution. Bioessays. 2007;29(4):392-402.

7. Archibald JM, Lane CE. Going, going, not quite gone: nucleomorphs as a case study in nuclear genome reduction. J Hered. 2009;100(5):582-90.

8. Zauner S, Fraunholz M, Wastl J, Penny S, Beaton M, Cavalier-Smith T, Maier UG, Douglas S. Chloroplast protein and centrosomal genes, a tRNA intron, and odd telomeres in an unusually compact eukaryotic genome, the cryptomonad nucleomorph. Proc Natl Acad Sci USA. 2000;97(1):200-5.

9. Douglas S, Zauner S, Fraunholz M, Beaton M, Penny S, Deng LT, Wu X Reith M, Cavalier-Smith T, Maier UG. The highly reduced genome of an enslaved algal nucleus. Nature. 2001;410(6832):1091-6.

10. Tanifuji G, Onodera NT, Wheeler TJ, Dlutek M, Donaher N, Archibald JM Complete nucleomorph genome sequence of the nonphotosynthetic alga Cryptomonas paramecium reveals a core nucleomorph gene set. Genome Biol Evol. 2011;3:44-54.

11. Moore CE, Curtis B, O Mills T, Tanifuji G, Archibald JM. Nucleomorph genome sequence of the cryptophyte alga Chroomonas mesostigmatica CCMP1168 reveals lineage-specific gene loss and genome complexity. Genome Biol Evol. 2012;4(11):1162-75.

12. Tanifuji G, Onodera NT, Brown MW, Curtis BA, Roger AJ, Ka-Shu Wong G, Melkonian M, Archibald JM. Nucleomorph and plastid genome sequences of the chlorarachniophyte Lotharella oceanica: convergent reductive evolution and frequent recombination in nucleomorph-bearing algae. BMC Genomics. 2014;15:374.

13. Gilson PR, Su V, Slamovits CH, Reith ME, Keeling PJ, McFadden GI. Complete nucleotide sequence of the chlorarachniophyte nucleomorph: nature's smallest nucleus. Proc Natl Acad Sci USA 2006;103(25):9566-71.

14. Lane CE, van den Heuvel K, Kozera C, Curtis BA, Parsons BJ, Bowman S, Archibald JM. Nucleomorph genome of Hemiselmis andersenii reveals complete intron loss and compaction as a driver of protein structure and function. Proc Natl Acad Sci USA. 2007;104(50):19908-13.

15. Jenuwein T, Allis CD. Translating the histone code. Science. 2001;293(5532):1074-80.

16. Huang H, Sabari BR, Garcia BA, Allis CD, Zhao Y. SnapShot: histone modifications. Cell. 2014;159(2):458-458.

17. Kouzarides T. Chromatin modifications and their function. Cell. 2007;128(4):693-705.

18. Postberg J, Forcob S, Chang WJ, Lipps HJ. The evolutionary history of histone $\mathrm{H} 3$ suggests a deep eukaryotic root of chromatin modifying mechanisms. BMC Evol Biol. 2010;10:259.

19. Li B, Carey M, Workman JL. The role of chromatin during transcription. Cell. 2007;128(4):707-19.

20. Wagner EJ, Carpenter PB. Understanding the language of Lys36 methylation at histone H3. Nat Rev Mol Cell Biol. 2012;13(2):115-126.

21. Lee JS, Shilatifard A. A site to remember: H3K36 methylation a mark for histone deacetylation. Mutat Res. 2007;618(1-2):130-134.

22. Reinberg D, Sims RJ. 3rd de FACTo nucleosome dynamics. J Biol Chem. 2006;281(33):23297-301.

23. Blasco MA. The epigenetic regulation of mammalian telomeres. Nat Rev Genet. 2007;8(4):299-309.

24. Stimpson KM, Sullivan BA. Epigenomics of centromere assembly and function. Curr Opin Cell Biol. 2010;22(6):772-80.

25. Lamb JC, Yu W, Han F, Birchler JA. Plant chromosomes from end to end: telomeres, heterochromatin and centromeres. Curr Opin Plant Biol. 2007;10(2):116-22. 
26. Wang F, Higgins JM. Histone modifications and mitosis: countermarks, landmarks, and bookmarks. Trends Cell Biol. 2013;23(4):175-84.

27. Buratowski S. Progression through the RNA polymerase II CTD cycle. Mol Cell. 2009;36(4):541-6.

28. Hsin JP, Manley JL. The RNA polymerase II CTD coordinates transcription and RNA processing. Genes Dev. 2012;26(19):2119-37.

29. Eddy SR. Accelerated Profile HMM Searches. PLoS Comput Biol. 2011;7(10):e1002195

30. Finn RD, Bateman A, Clements J, Coggill P, Eberhardt RY, Eddy SR, Heger A, Hetherington K, Holm L, Mistry J, Sonnhammer EL, Tate J, Punta M. Pfam: the protein families database. Nucleic Acids Res. 2014;42(Database issue):D222-230.

31. Altschul SF, Madden TL, Schäffer AA, Zhang J, Zhang Z, Miller W Lipman DJ. Gapped BLAST and PSI-BLAST: a new generation of protein database search programs. Nucleic Acids Res. 1997;25(17):3389-3402.

32. Hirakawa Y, Burki F, Keeling PJ. Nucleus- and nucleomorph-targeted histone proteins in a chlorarachniophyte alga. Mol Microbiol. 2011;80(6): 1439-49.

33. Bannister AJ, Zegerman P, Partridge JF, Miska EA, Thomas JO Allshire RC, Kouzarides T. Selective recognition of methylated lysine 9 on histone H3 by the HP1 chromo domain. Nature. 2001;410(6824):120-4.

34. Lachner M, O'Carroll D, Rea S, Mechtler K, Jenuwein T. Methylation of histone $\mathrm{H} 3$ lysine 9 creates a binding site for HP1 proteins. Nature. 2001:410(6824):116-20.

35. Schotta G, Lachner M, Sarma K, Ebert A, Sengupta R, Reuter G, Reinberg D, Jenuwein T. A silencing pathway to induce H3-K9 and H4-K20 trimethylation at constitutive heterochromatin. Genes Dev. 2004;18(11):1251-62.

36. Balakrishnan L, Milavetz B. Decoding the histone $\mathrm{H} 4$ lysine 20 methylation mark. Crit Rev Biochem Mol Biol. 2010;45(5):440-52.

37. Daujat S, Weiss T, Mohn F, Lange UC, Ziegler-Birling C, Zeissler U, Lappe M, Schübeler D, Torres-Padilla ME, Schneider R. H3K64 trimethylation marks heterochromatin and is dynamically remodeled during developmental reprogramming. Nat Struct Mol Biol. 2009;16(7): 777-81.

38. Zheng B, Chen X. Dynamics of histone $\mathrm{H} 3$ lysine 27 trimethylation in plant development. Curr Opin Plant Biol. 2011;14(2):123-9.

39. Simon JA, Kingston RE. Occupying chromatin: Polycomb mechanisms for getting to genomic targets, stopping transcriptional traffic, and staying put. Mol Cell. 2013;49(5):808-24.

40. Filion GJ, van Bemmel JG, Braunschweig U, Talhout W, Kind J, Ward LD, Brugman W, de Castro IJ, Kerkhoven RM, Bussemaker HJ, van Steensel B. Systematic protein location mapping reveals five principal chromatin types in Drosophila, cells. Cell. 2010;143(2):212-24.

41. Riddle NC, Minoda A, Kharchenko PV, Alekseyenko AA, Schwartz YB, Tolstorukov MY, Gorchakov AA, Jaffe JD, Kennedy C, Linder-Basso D, Peach SE, Shanower G, Zheng H, Kuroda MI, Pirrotta V, Park PJ, Elgin SC, Karpen GH. Plasticity in patterns of histone modifications and chromosomal proteins in Drosophila heterochromatin. Genome Res. 2011;21(2):147-63.

42. Kelly AE, Ghenoiu C, Xue JZ, Zierhut C, Kimura H, Funabiki H. Survivin reads phosphorylated histone $\mathrm{H} 3$ threonine 3 to activate the mitotic kinase Aurora B. Science. 2010;330(6001):235-9.

43. Wang F, Dai J, Daum JR, Niedzialkowska E, Banerjee B, Stukenberg PT, Gorbsky GJ, Higgins JM. Histone H3 Thr-3 phosphorylation by Haspin positions Aurora B at centromeres in mitosis. Science. 2010;330(6001): 231-5.

44. Polioudaki H, Markaki Y, Kourmouli N, Dialynas G, Theodoropoulos PA, Singh PB, Georgatos SD. Mitotic phosphorylation of histone $\mathrm{H} 3$ at threonine 3. FEBS Lett. 2004;560(1-3):39-44

45. Johansen KM, Johansen J. Regulation of chromatin structure by histone H3S10 phosphorylation. Chromosome Res. 2006;14(4):393-404.

46. Hendzel MJ, Wei Y, Mancini MA, Van Hooser A, Ranalli T, Brinkley BR, Bazett-Jones DP, Allis CD. Mitosis-specific phosphorylation of histone $\mathrm{H} 3$ initiates primarily within pericentromeric heterochromatin during G2 and spreads in an ordered fashion coincident with mitotic chromosome condensation. Chromosoma. 1997;106(6):348-60.

47. Wei Y, Mizzen CA, Cook RG, Gorovsky MA, Allis CD. Phosphorylation of histone $\mathrm{H} 3$ at serine 10 is correlated with chromosome condensation during mitosis and meiosis in Tetrahymena. Proc Natl Acad Sci USA 1998;95(13):7480-4.
48. Wei Y, Yu L, Bowen J, Gorovsky MA, Allis CD. Phosphorylation of histone $\mathrm{H} 3$ is required for proper chromosome condensation and segregation. Cell. 1999;97(1):99-109.

49. Barber CM, Turner FB, Wang Y, Hagstrom K, Taverna SD, Mollah S, Ueberheide B, Meyer BJ, Hunt DF, Cheung P, Allis CD. The enhancement of histone $\mathrm{H} 4$ and $\mathrm{H} 2 \mathrm{~A}$ serine 1 phosphorylation during mitosis and S-phase is evolutionarily conserved. Chromosoma. 2004;112(7):360-71.

50. Metzger E, Yin N, Wissmann M, Kunowska N, Fischer K, Friedrichs N, Patnaik D, Higgins JM, Potier N, Scheidtmann KH, Buettner R, Schüle R. Phosphorylation of histone $\mathrm{H} 3$ at threonine 11 establishes a novel chromatin mark for transcriptional regulation. Nat Cell Biol. 2008;10(1): $53-60$

51. Hammond SL, Byrum SD, Namjoshi S, Graves HK, Dennehey BK, Tackett AJ, Tyler JK. Mitotic phosphorylation of histone $\mathrm{H} 3$ threonine 80 Cell Cycle. 2014;13(3):440-52

52. Preuss $U$, Landsberg $\mathrm{G}$, Scheidtmann KH. Novel mitosis-specific phosphorylation of histone $\mathrm{H} 3$ at Thr11 mediated by DIk/ZIP kinase. Nucleic Acids Res. 2003;31(3):878-85.

53. Yamagishi $Y$, Honda $T$, Tanno $Y$, Watanabe $Y$. Two histone marks establish the inner centromere and chromosome bi-orientation. 330 2010;6001:239-43.

54. Sawicka A, Hartl D, Goiser M, Pusch O, Stocsits RR, Tamir IM, Mechtler K Seiser C. H3S28 phosphorylation is a hallmark of the transcriptional response to cellular stress. Genome Res. 2014;24(11):1808-20.

55. Sun JM, Chen HY, Espino PS, Davie JR. Phosphorylated serine 28 of histone $\mathrm{H} 3$ is associated with destabilized nucleosomes in transcribed chromatin. Nucleic Acids Res. 2007;35(19):6640-47.

56. Orphanides G, LeRoy G, Chang CH, Luse DS, Reinberg D. FACT, a factor that facilitates transcript elongation through nucleosomes. Cell. 1998;92(1):105-16.

57. Belotserkovskaya R, Oh S, Bondarenko VA, Orphanides G, Studitsky VM, Reinberg D. FACT facilitates transcription-dependent nucleosome alteration. Science. 2003;301(5636):1090-93.

58. Pavri R, Zhu B, Li G, Trojer P, Mandal S, Shilatifard A, Reinberg D. Histone $\mathrm{H} 2 \mathrm{~B}$ monoubiquitination functions cooperatively with FACT to regulate elongation by RNA polymerase II. Cell. 2006;125(4):703-17.

59. Zhu B, Zheng Y, Pham AD, Mandal SS, Erdjument-Bromage $H$, Tempst $P$, Reinberg D. Monoubiquitination of human histone $\mathrm{H} 2 \mathrm{~B}$ : the factors involved and their roles in $\mathrm{HOX}$ gene regulation. Mol Cell. 2005;20(4):601-11.

60. Bannister AJ, Schneider R, Myers FA, Thorne AW, Crane-Robinson C, Kouzarides T. Spatial distribution of di- and tri-methyl lysine 36 of histone $\mathrm{H} 3$ at active genes. J Biol Chem. 2005;280(18):17732-36.

61. Li J, Moazed D, Gygi SP. Association of the histone methyltransferase Set2 with RNA polymerase II plays a role in transcription elongation. J Biol Chem. 2002;277(51):49383-88.

62. Carrozza MJ, Li B, Florens L, Suganuma T, Swanson SK, Lee KK, Shia WJ, Anderson S, Yates J, Washburn MP, Workman JL. Histone H3 methylation by Set2 directs deacetylation of coding regions by Rpd3S to suppress spurious intragenic transcription. Cell. 2005;123(4):581-92.

63. Joshi AA, Struhl K. Eaf3 chromodomain interaction with methylated H3-K36 links histone deacetylation to Pol II elongation. Mol Cell. 2005;20(6):971-78

64. Keogh MC, Kurdistani SK, Morris SA, Ahn SH, Podolny V, Collins SR, Schuldiner M, Chin K, Punna T, Thompson NJ, Boone C, Emili A, Weissman JS, Hughes TR, Strahl BD, Grunstein M, Greenblatt JF, Buratowski S, Krogan NJ. Cotranscriptional set2 methylation of histone H3 lysine 36 recruits a repressive Rpd3 complex. Cell. 2005;123(4): 593-605.

65. Kaplan CD, Laprade L, Winston F. Transcription elongation factors repress transcription initiation from cryptic sites. Science. 2003;301(5636):1096-99.

66. Nguyen AT, Zhang Y. The diverse functions of Dot1 and H3K79 methylation. Genes Dev. 2011;25(13):1345-58.

67. van Leeuwen F, Gafken PR, Gottschling DE. Dot1p modulates silencing in yeast by methylation of the nucleosome core. Cell. 2002;109(6): 745-56

68. Vakoc CR, Sachdeva MM, Wang H, Blobel GA. Profile of histone lysine methylation across transcribed mammalian chromatin. Mol Cell Biol. 2006;26(24):9185-95. 
69. Steger DJ, Lefterova MI, Ying L, Stonestrom AJ, Schupp M, Zhuo D, Vakoc AL, Kim JE, Chen J, Lazar MA, Blobel GA, Vakoc CR. DOT1L/KMT4 recruitment and $\mathrm{H} 3 \mathrm{~K} 79$ methylation are ubiquitously coupled with gene transcription in mammalian cells. Mol Cell Biol. 2008;28(8):2825-39.

70. Kolasinska-Zwierz P, Down T, Latorre I, Liu T, Liu XS, Ahringer J. Differential chromatin marking of introns and expressed exons by H3K36me3. Nat Genet. 2009;41(3):376-81.

71. Schwartz S, Meshorer E, Ast G. Chromatin organization marks exon-intron structure. Nat Struct Mol Biol. 2009;16(9):990-5.

72. Spies N, Nielsen CB, Padgett RA, Burge CB. Biased chromatin signatures around polyadenylation sites and exons. Mol Cell. 2009;36(2):245-54.

73. Luco RF, Pan Q, Tominaga K, Blencowe BJ, Pereira-Smith OM, Misteli T. Regulation of alternative splicing by histone modifications. Science. 2010;327(5968):996-1000.

74. Kim S, Kim H, Fong N, Erickson B, Bentley DL. Pre-mRNA splicing is a determinant of histone H3K36 methylation. Proc Natl Acad Sci USA. 2011;108(33):13564-69.

75. de Almeida SF, Grosso AR, Koch F, Fenouil R, Carvalho S, Andrade J, Levezinho H, Gut M, Eick D, Gut I, Andrau JC, Ferrier P, Carmo-Fonseca M. Splicing enhances recruitment of methyltransferase HYPB/Setd2 and methylation of histone H3 Lys36. Nat Struct Mol Biol. 2011;18(9):977-983.

76. Bernstein BE, Humphrey EL, Erlich RL, Schneider R, Bouman P, Liu JS, Kouzarides T, Schreiber SL. Methylation of histone H3 Lys 4 in coding regions of active genes. Proc Natl Acad Sci USA. 2002;99(13):8695-700.

77. Shilatifard A. The COMPASS family of histone H3K4 methylases: mechanisms of regulation in development and disease pathogenesis. Annu Rev Biochem. 2012;81:65-95.

78. Guccione E, Bassi C, Casadio F, Martinato F, Cesaroni M, Schuchlautz H, Lüscher B, Amati B. Methylation of histone H3R2 by PRMT6 and H3K4 by an MLL complex are mutually exclusive. Nature. 2007;449(7164):933-7.

79. Kirmizis A, Santos-Rosa H, Penkett CJ, Singer MA, Vermeulen M, Mann M, Bähler J, Green RD, Kouzarides T. Arginine methylation at histone H3R2 controls deposition of H3K4 trimethylation. Nature. 2007;449(7164):928-32.

80. Yuan CC, Matthews AG, Jin Y, Chen CF, Chapman BA, Ohsumi TK, Glass KC, Kutateladze TG, Borowsky ML, Struhl K, Oettinger MA. Histone H3R2 symmetric dimethylation and histone H3K4 trimethylation are tightly correlated in eukaryotic genomes. Cell Rep. 2012;1(2):83-90.

81. Yue M, Li Q, Zhang Y, Zhao Y, Zhang Z, Bao S. Histone H4R3 methylation catalyzed by SKB1/PRMT5 is required for maintaining shoot apical meristem. PLoS ONE. 2013;8(12):e83258.

82. Dhar SS, Lee SH, Kan PY, Voigt P, Ma L, Shi X, Reinberg D, Lee MG. Trans-tail regulation of MLL4-catalyzed H3K4 methylation by H4R3 symmetric dimethylation is mediated by a tandem PHD of MLL4. Genes Dev. 2012;26(24):2749-62.

83. Metzger E, Imhof A, Patel D, Kahl P, Hoffmeyer K, Friedrichs N, Müller JM, Greschik H, Kirfel J, Ji S, Kunowska N, Beisenherz-Huss C, Günther T, Buettner R, Schl̈e R. Phosphorylation of histone H3T6 by

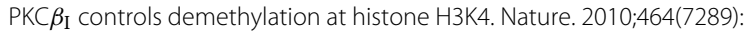
792-6.

84. Xie W, Song C, Young NL, Sperling AS, Xu F, Sridharan R, Conway AE, Garcia BA, Plath K, Clark AT, Grunstein M. Histone h3 lysine 56 acetylation is linked to the core transcriptional network in human embryonic stem cells. Mol Cell. 2009;33(4):417-27.

85. Xu F, Zhang K, Grunstein M. Acetylation in histone $\mathrm{H} 3$ globular domain regulates gene expression in yeast. Cell. 2005;121(3):375-85.

86. Rufiange A, Jacques PE, Bhat W, Robert F, Nourani A. Genome-wide replication-independent histone $\mathrm{H} 3$ exchange occurs predominantly at promoters and implicates $\mathrm{H} 3 \mathrm{~K} 56$ acetylation and Asf1. Mol Cell. 2007;27(3):393-405

87. Yang X, Yu W, Shi L, Sun L, Liang J, Yi X, Li Q, Zhang Y, Yang F, Han X, Zhang D, Yang J, Yao Z, Shang Y. HAT4, a Golgi apparatus-anchored B-type histone acetyltransferase, acetylates free histone $\mathrm{H} 4$ and facilitates chromatin assembly. Mol Cell. 2011;44(1):39-50.

88. Eick D, Geyer M. The RNA polymerase II carboxy-terminal domain (CTD) code. Chem Rev. 2013;113(11):8456-90.

89. Yang C, Stiller JW. Evolutionary diversity and taxon-specific modifications of the RNA polymerase II C-terminal domain. Proc Natl Acad Sci USA. 2014;111(16):5920-25.
90. Allison LA, Moyle M, Shales M, Ingles CJ. Extensive homology among the largest subunits of eukaryotic and prokaryotic RNA polymerases. Cell. 1985;42(2):599-610.

91. Tanifuji G, Onodera NT, Moore CE, Archibald JM. Reduced nuclear genomes maintain high gene transcription levels. Mol Biol Evol. 2014;31(3):625-35.

92. Dion MF, Altschuler SJ, Wu LF, Rando OJ. Genomic characterization reveals a simple histone $\mathrm{H} 4$ acetylation code. Proc Natl Acad Sci USA. 2005;102(15):5501-06.

93. Martin AM, Pouchnik DJ, Walker JL, Wyrick JJ. Redundant roles for histone $\mathrm{H} 3 \mathrm{~N}$-terminal lysine residues in subtelomeric gene repression in Saccharomyces cerevisiae. Genetics. 2004;167(3):1123-32.

94. Dillon SC, Zhang X, Trievel RC, Cheng X. The SET-domain protein superfamily: protein lysine methyltransferases. Genome Biol. 2005;6(8): 227.

95. Marmorstein R, Zhou MM. Writers and readers of histone acetylation: structure, mechanism, and inhibition. Cold Spring Harb Perspect Biol. 2014;6(7):a018762.

96. Vaquero A. The conserved role of sirtuins in chromatin regulation. Int J Dev Biol. 2009;53(2-3):303-22.

97. Sanchez R, Meslamani J, Zhou MM. The bromodomain: from epigenome reader to druggable target. Biochim Biophys Acta. 2014;1839(8):676-85.

98. Blus BJ, Wiggins K, Khorasanizadeh S. Epigenetic virtues of chromodomains. Crit Rev Biochem Mol Biol. 2011;46(6):507-26.

99. Sanchez R, Zhou MM. The PHD finger: a versatile epigenome reader. Trends Biochem Sci. 2011;36(7):364-72.

100. Takeuchi T, Watanabe Y, Takano-Shimizu T, Kondo S. Roles of jumonji and jumonji family genes in chromatin regulation and development. Dev Dyn. 2006;235(9):2449-59.

101. Burgess RJ, Zhang Z. Histone chaperones in nucleosome assembly and human disease. Nat Struct Mol Biol. 2013;20(1):14-22.

102. Edgar RC. MUSCLE: multiple sequence alignment with high accuracy and high throughput. Nucleic Acids Res. 2004;32(5):1792-97.

103. Waterhouse AM, Procter JB, Martin DM, Clamp M, Barton GJ. Jalview Version 2-a multiple sequence alignment editor and analysis workbench. Bioinformatics. 2009;25(9):1189-91.

104. Hopkins JF, Spencer DF, Laboissiere S, Neilson JA, Eveleigh RJ, Durnford DG, Gray MW, Archibald JM. Proteomics reveals plastid- and periplastid-targeted proteins in the chlorarachniophyte alga Bigelowiella natans. Genome Biol Evol. 2012;4(12):1391-1406.

105. Keeling PJ, Burki F, Wilcox HM, Allam B, Allen EE, Amaral-Zettler LA, Armbrust EV, Archibald JM, Bharti AK, Bell CJ, Beszteri B, Bidle KD, Cameron CT, Campbell L, Caron DA, Cattolico RA, Collier JL, Coyne K, Davy SK, Deschamps P, Dyhrman ST, Edvardsen B, Gates RD, Gobler CJ, Greenwood SJ, Guida SM, Jacobi JL, Jakobsen KS, James ER, Jenkins B, John U, Johnson MD, Juhl AR, Kamp A, Katz LA, Kiene R, Kudryavtsev A, Leander BS, Lin S, Lovejoy C, Lynn D, Marchetti A, McManus G, Nedelcu AM, Menden-Deuer S, Miceli C, Mock T, Montresor M, Moran MA, Murray S, Nadathur G, Nagai S, Ngam PB, Palenik B, Pawlowski J, Petroni G, Piganeau G, Posewitz MC, Rengefors K, Romano G, Rumpho ME, Rynearson T, Schilling KB, Schroeder DC, Simpson AG, Slamovits CH, Smith DR, Smith GJ, Smith SR, Sosik HM, Stief P, Theriot E, Twary SN, Umale PE, Vaulot D, Wawrik B, Wheeler GL, Wilson WH, Xu Y, Zingone A, Worden AZ. The Marine Microbial Eukaryote Transcriptome Sequencing Project (MMETSP): illuminating the functional diversity of eukaryotic life in the oceans through transcriptome sequencing. PLoS Biol. 2014;12(6):e1001889. 